\title{
Quantifying the discharge forecast uncertainty by different approaches to probabilistic quantitative precipitation forecast
}

\author{
T. Diomede, C. Marsigli, F. Nerozzi, T. Paccagnella, and A. Montani \\ Regional Hydro-Meteorological Service ARPA-SIM, Bologna, Italy \\ Received: 10 November 2005 - Revised: 13 January 2006 - Accepted: 17 January 2006 - Published: 28 February 2006
}

\begin{abstract}
A probabilistic approach to flood prediction over the Reno river basin, a medium-sized catchment in Northern Italy, has been tested using two different meteorological ensemble systems. The future precipitation scenarios are provided either by an analogue-based technique (statistical approach) or by a limited-area ensemble prediction system (dynamical approach), then used as different inputs to a distributed rainfall-runoff model. The ensemble of possible future flows so generated allows to convey a quantification of uncertainty about the discharge forecast. The probabilistic discharge forecasts, based on the precipitation forecast provided by the two ensembles, are then compared to the deterministic one obtained by the rainfall-runoff model fed on precipitation input provided by a non-hydrostatic meteorological model, run at $7 \mathrm{~km}$ of horizontal resolution. For this case study, the dynamical approach appears to be more feasible in providing useful discharge ensemble forecast than the statistical one, because the observed large spread among members obtained with the analogue method makes difficult to issue real-time flood warnings.
\end{abstract}

\section{Introduction}

The need to deal with uncertainties in hydrological model predictions has been widely recognised in recent years, in such a way that the classical perception of a single-valued forecast river flow should be enhanced by its associated uncertainty, especially when real-time flood warnings are issued. In particular, at a medium-sized basin scale, a probabilistic approach to quantitative precipitation forecasts (QPFs), used to force hydrological models, seems to be indispensable to obtain different future discharge scenarios, enabling to manage the flood counting for the variability of phenomena and the uncertainty associated with an hydrologic forecast. In this work, the use of uncertainty in hydrolog- ical model predictions is related with the problem to integrate meteorological forecast uncertainty into a hydrological model capable to propagate such into hydrological forecast and warning uncertainty, adapting existing concepts of probabilistic forecast products from atmospheric modelling to flood forecasting.

In the last 15 years, the meteorological community has made a larger and larger use of ensemble prediction systems to assess the uncertainty involved in forecasting precipitation in time and space and to gain additional information on the characteristics of possible events. Nowadays, it looks more and more feasible to use meteorological ensemble systems so as force hydrological forecasts in order to improve both the accuracy of forecasts and the reliability of uncertainty estimates.

In this work, ensembles of future precipitation scenarios have been provided by an analogue-based technique and by COSMO-LEPS (the Limited-area Ensemble Prediction System, developed within the COnsortium for Small-scale MOdelling; Marsigli et al., 2005), then used as different inputs to the TOPKAPI (TOPographic Kinematic Approximation and Integration) model (Todini and Ciarapica, 2001), a physically based rainfall-runoff model, conveying a quantification of uncertainty about the discharge prediction occurred over the Reno river basin, a catchment in Northern Italy whose dimension is $4930 \mathrm{~km}^{2}$.

The methodology has been tested for two flood events occurred on 15-20 November 2002 and 6-11 November 2003. Making the assumption of a perfect forecast hydrological model, the discharge calculated using the precipitation recorded by the available rain-gauges has been taken as reference for the discharge predictions based on analogues and COSMO-LEPS forecasts. The forecasts are referred to Casalecchio Chiusa, near Bologna, the closure section of the mountainous catchment covering $1051 \mathrm{~km}^{2}$. 


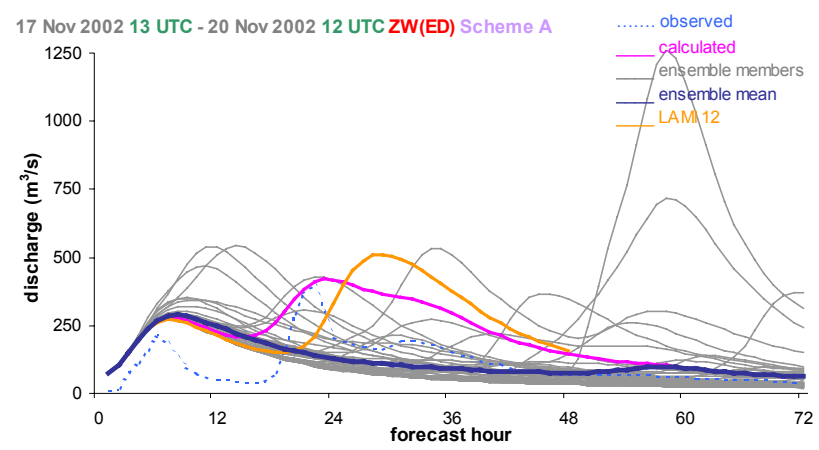

Fig. 1. Ensemble of discharge predictions (grey lines) provided with a lead-time of $24 \mathrm{~h}$ by the analogue-based scheme A for the 15-20 November 2002 events (the ensemble mean is displayed by a thick dark blue line). The ensemble is compared to the discharge prediction based on the precipitation forecast provided by LM (orange line). It is also displayed the observed discharge (dotted blue line) and the calculated one (fuchsia line), obtained by the model fed with the rain-gauge measures spatially distributed by the Block-Kriging technique.

\section{The ensemble of discharge forecasts based on ana- logues}

The analogue method is based on a search for past situations similar to the day at hand (analogues). The similarity is assessed in terms of synoptic circulation pattern over Western Europe and East Atlantic, as defined by the combination of geopotential height $(\mathrm{Z})$ at $500 \mathrm{hPa}$ and vertical velocity (W) at $700 \mathrm{hPa}$. The archive of similar past situations contains analyses and forecasts of these variables as provided by ECMWF (European Centre for Medium-range Weather Forecasts). Both $\mathrm{Z}$ and $\mathrm{W}$ are available every day at 12:00 UTC for the period 1990-2003, over the area $10^{\circ} \mathrm{W}-$ $20^{\circ} \mathrm{E}, 30^{\circ} \mathrm{N}-60^{\circ} \mathrm{N}$, with a $0.5^{\circ}$ grid spacing.

According to the euclidean distance (hereafter ED) similarity criterion, a fifty-member subset of these analogues is singled out and the corresponding rainfall measurements, recorded for the next $72 \mathrm{~h}$ by the raingauges spread over the Reno river basin, are treated as the precipitation forecasts (Diomede et al., 2003). These fifty rain time-series represent the probabilistic inputs for the TOPKAPI model, thus generating an ensemble of discharge forecasts.

The forecasts obtained via this approach (hereafter, scheme A) are compared with those provided by other two methods (hereafter, scheme B and C), based on the proposal of Obled et al. (2002). The three approaches can be summarized as follows. In the scheme $A$, each current day $D_{c}$ and each past analogue day $D_{p}$ is characterised by ECMWF analyses at 12:00 UTC of day D and day D-1 and the precipitation forecast is obtained by the next $72 \mathrm{~h}$ of historical rain-gauge recordings starting from 13:00 UTC of day $D_{p}$. In the scheme $B$, the days $D_{c}$ and $D_{p}$ are characterised by ECMWF analyses at 12:00 UTC of day D and corresponding model forecasts at $+24,+48$ and $+72 \mathrm{~h}$. For each of the three different forecast times, the related precipitation forecast is obtained by

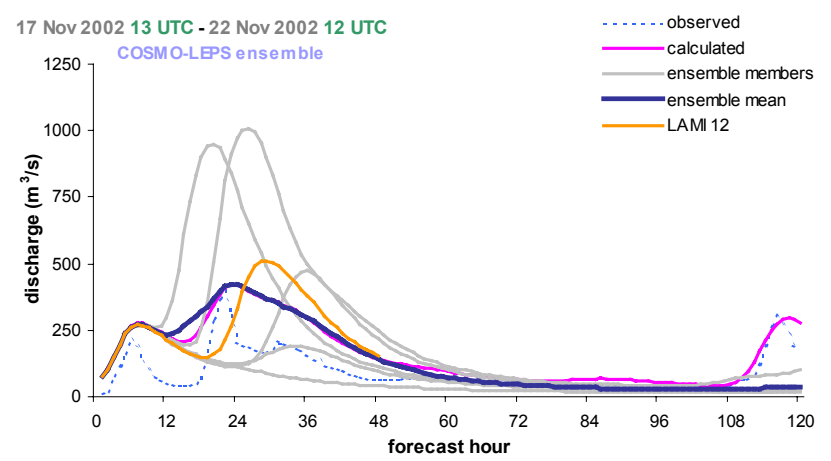

Fig. 2. Ensemble of discharge predictions based on the precipitation forecasts provided with a lead-time of $24 \mathrm{~h}$ by the COSMO-LEPS system for the 15-20 November 2002 events. It is also displayed the ensemble mean (thick dark blue line).

the $24 \mathrm{~h}$ historical rain-gauge recordings characterising the corresponding past analogue day up to achieve the $72 \mathrm{~h}$ raintime series. The scheme $\mathrm{C}$ differs from the scheme $\mathrm{B}$ only in the definition of day $D_{p}$, since it is characterised by ECMWF analyses at 12:00 UTC of day $D_{p}$ and day $D_{p}+1$

For the selected case studies, the discharge predictions supplied by the three approaches are quite good for the first 24 forecast hours (displayed in Fig. 1 only for the 15-20 November 2002 event, scheme A), showing a decay of performance with increasing lead-time. This drawback can be partially overcome updating the search for analogues every $24 \mathrm{~h}$ by means of the meteorological variable forecast provided by a numerical model. This solution characterises both schemes $\mathrm{B}$ and $\mathrm{C}$, which have provided rather similar outcomes.

A shortcoming of the analogue method is that the available historical meteo-hydrological archive is not large enough to reproduce a reliable scenario in case of extreme events.

\section{The ensemble of discharge forecasts based on COSMO-LEPS}

The COSMO-LEPS methodology attempts to combine the benefits of the probabilistic approach with the highresolution capabilities of the Limited Area Model integrations. COSMO-LEPS is a mesoscale ensemble prediction system based on a few integrations of the limited-area model Lokal Modell (LM). The system has been developed and maintained by ARPA-SIM and is running regularly at ECMWF since November 2002 thanks to the efforts of the COSMO Consortium. The COSMO-LEPS ensemble members are differentiated mainly in the boundary conditions by which they are driven: the LM runs are nested on some selected members of ECMWF Ensemble Prediction System (EPS), chosen by means of an ensemble-size reduction technique based on a Cluster Analysis algorithm, at a 120-h forecast range and with a horizontal resolution of about $10 \mathrm{~km}$ and 32 layers in the vertical. The system has been recently 
updated to the 10-member version, but at the time of the experiments here described the old 5-member suite was operational (Marsigli et al., 2005).

The spatial grid resolution of COSMO-LEPS system is comparable to that retrieved by applying the Thiessen polygons method to the available raingauge network. Furthermore, for the selected watershed size, the COSMO-LEPS spatial and temporal resolution may likely be able to resolve the rainfall field at the basin scale and therefore the model outputs may be directly coupled to a hydrological model, without the need to use some kind of stochastic procedure for rainfall downscaling, as indicated by Ferraris et al. (2002) and Siccardi et al. (2005). In the direct coupling here adopted, all members are considered equally probable.

The discharge predictions based on the COSMO-LEPS ensemble precipitation forecasts yield very different results over the two case studies. For the 15-20 November 2002 event, the forecast is fairly accurate, especially for the shorter lead-time, when the ensemble mean perfectly reproduces the calculated discharge (Fig. 2). On the other hand, for the 611 November 2003 event the calculated river flow is heavily underestimated at each lead-time by all the ensemble members (not shown); a further investigation suggested that this is principally due to the lack of spread in the global ensemble over the region of interest.

\section{Conclusions}

At a medium-sized basin scale, quantifying precipitation which is likely to occur in the next few days is still a challenge, because uncertainty is considerable and reliable numerical deterministic precipitation forecasts to drive a hydrological model are very difficult to achieve. Therefore, the uncertainty in precipitation forecasts must be quantified in order to provide informative hydrologic forecasts: ensembles are a convenient method of handling uncertainty, since information about forecast uncertainty could be derived from the dispersion of ensemble members.

An ensemble approach has been applied as forecast methodology, using multiple precipitation forecast scenarios to generate a discharge forecast ensemble by the execution of several hydrological model runs. The case studies here analysed claim that the dynamical approach appears to be more feasible in order to provide discharge ensemble forecast than the statistical one, because the observed large spread among members obtained with the analogue method makes difficult to issue real-time flood warnings. This large spread, similar in size to the entire climatological variability, is principally due to the limitation in archived data availability. On the other hand, in some cases, as highlighted in the 6-11 November 2003 event, COSMO-LEPS did not manage to provide a sufficient amount of spread among members, not allowing to forecast the occurrence of the extreme event.
Finally, the probabilistic information on future hydrological flows, obtained by the ensemble forecast methods, should be considered as complementary, and not alternative, to the deterministic one based on the precipitation forecast provided by the limited-area model Lokal Modell.

Within a theoretical framework, a more correct approach to assess the forecast uncertainty should be performed using statistical methods (such as confidence intervals of the quantity of interest) to compute the probability associated to the prediction. However, in this work, the forecast expressed in terms of quantiles should be adequate for the analogue technique due to the size of the ensemble, while a confidence interval built on few members, like the case of COSMOLEPS system, could lack in significance because of the large spread among members. Thus, the spread of the ensemble has been employed to convey the forecast uncertainty, since it is a common practice and the research is addressed to provide different scenarios of possible future streamflow rather than a probabilistic information based on a confidence interval of the expected future discharge.

For the future, more studies and further developments are needed to generalise these results and to improve the ensemble forecasting approach for hydrological predictions.

Edited by: V. Kotroni and K. Lagouvardos

Reviewed by: anonymous referee

\section{References}

Diomede, T., Nerozzi, F., Paccagnella, T., Tibaldi, S., and Todini, E.: Using LAM and analogue-based rainfall prediction ensembles to reduce flood forecasting uncertainty, Proceedings of the 5th EGS Plinius Conference, PLC5-A-00018, 2003.

Ferraris, L., Rudari, R., and Siccardi, F.: The uncertainty in the prediction of flash floods in the northern mediterranean environment, J. Hydrometeorol., 3, 714-727, 2002.

Marsigli, C., Boccanera, F., Montani, A., and Paccagnella, T.: The COSMO-LEPS mesoscale ensemble system: validation of the methodology and verification, Nonlin. Processes Geophys., 12, 527-536, 2005.

Obled, C., Bontron, G., and Garçon, R.: Quantitative precipitation forecasts: a statistical adaptation of model outputs through an analogues sorting approach, Atmos. Res., 63, 303-324, 2002.

Siccardi, F., Boni, G., Ferraris, L., and Rudari, R.: A hydrometeorological approach for probabilistic flood forecast, J. Geophys. Res., 110, D05101, doi:10.1029/2004JD005314, 2005.

Todini, E. and Ciarapica, L.: The TOPKAPI model, Mathematical Models of Large W atershed Hydrology, Chapter 12, edited by: Singh, V. P., Frevert, D. K., and Meyer, S. P., Water Resources Publications, Littleton, Colorado, 2001. 